\title{
Development of a high-pressure option for neutron scattering experiments at low temperatures and high magnetic fields at the MLZ
}

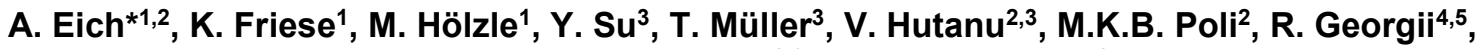 \\ L. Beddrich ${ }^{4,5}$, M. Meven ${ }^{2,3}$ and A. Grzechnik ${ }^{2}$ \\ ${ }^{I}$ Jülich Centre for Neutron Science-2/Peter Grünberg-Institute-4, Forschungszentrum Jülich GmbH, 52425 Jülich, Germany \\ ${ }^{2}$ Institute of Crystallography, RWTH Aachen University, 52056 Aachen, Germany \\ ${ }^{3}$ Jülich Centre for Neutron Science at Heinz Maier-Leibnitz-Zentrum, Forschungszentrum Jülich GmbH, 85747 Garching, Germany \\ ${ }^{4}$ Heinz Maier-Leibnitz-Zentrum, Technische Universität München, 85747 Garching, Germany \\ ${ }^{5}$ Physik-Department E21, Technische Universität München, 85748 Garching, Germany \\ *a.eich@fz-juelich.de
}

To study the effect of high pressure on any sample property, suitable pressure devices are a fundamental requirement. Their design has to be tailored to the experimental demands regarding the intended pressure, the employed instrumentation and the expected scientific results. Our work presents the development of high pressure cells for neutron scattering on polycrystalline and single-crystalline samples at low temperatures and with applied magnetic fields.

One of the most common devices for high-pressure neutron experiments is the clamp cell [1], where the pressure is applied ex situ and which can be used independently in various setups. Our cell design [2] has been specifically developed for neutron scattering experiments at low temperatures in the closed-cycle cryostats on the instruments DNS (diffuse scattering neutron spectrometer), MIRA (cold three axes spectrometer), and POLI (polarized hot neutron diffractometer) at the Heinz Maier-Leibnitz Zentrum (MLZ) in Garching, Germany. Two variants of the compact monobloc cell (Fig. 1) were produced, one from CuBe alloy and from NiCrAl "Russian Alloy", working up to about $1.1 \mathrm{GPa}$ and $1.5 \mathrm{GPa}$, respectively. The low paramagnetic moment of both alloys allows also measurements of magnetic properties.

First tests of the cell with neutron radiation were performed to calibrate the load/pressure-curve of the CuBe cell (up to $1.15 \mathrm{GPa}$ ) (at POLI), to estimate its neutron absorption and background (at MIRA), and to measure magnetic reflections (at MIRA). In addition, the thermal response in the cryostat of DNS was measured, and the experimental findings were complemented by simulations.

Ultimately, these cells are intended as standard cells for high pressure measurements on different instruments at MLZ suitable for all available magnets and cryostats down to $1.5 \mathrm{~K}$. Further tests under various conditions (temperature, pressure, magnetic field) as well as simulations are planned for both cells in the near future. The results will help both to establish the present cells and to optimise the design of subsequent cells to achieve higher pressures, to fit into smaller cryostats and to enable neutron-independent pressure calibration.

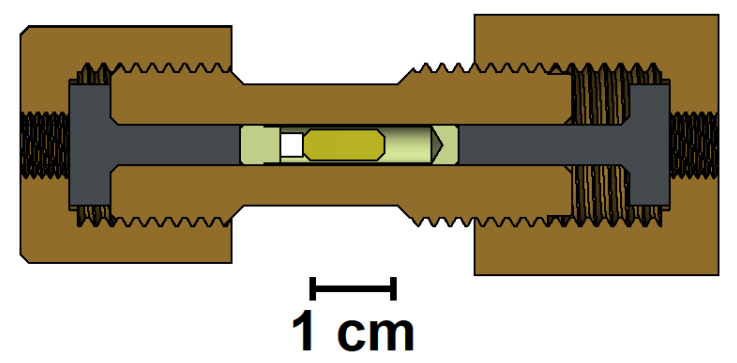

Figure 1. Schematic drawing of the clamp cell.

[1] Klotz, S. (2013). Techniques in High Pressure Neutron Scattering. CRC Press.

[2] Eich, A., Hölzle, M., Su, Y., Hutanu, V., Georgii, R., Beddrich, L. \& Grzechnik, A. (2020). High Press. Res. 41[1], 88-96.

Keywords: high pressure; neutron scattering; clamp cells

This work was supported by the Bundesministerium für Bildung und Forschung (BMBF) [grant number 05K19PA2] and by the Deutsche Forschungsgemeinschaft (DFG) [grant number GE971/5-2]. 